\title{
THE K-BAND HUBBLE DIAGRAM FOR THE BRIGHTEST CLUSTER GALAXIES: A TEST OF GALAXY FORMATION MODELS.
}

\author{
A. ARAGÓN-SALAMANCA \\ Institute of Astronomy \\ Madingley Road, Cambridge CB3 OHA, England \\ C.M. BAUGH \\ Department of Physics \\ Science Laboratories, South Road, Durham DH1 3LE, England \\ AND \\ G. KAUFFMANN \\ Max-Plank-Institut für Astrophysik \\ D-85740 Garching bei München, Germany
}

We analyze the K-band Hubble diagram for a sample of brightest cluster galaxies (BCGs) in the redshift range $0<z<1$. We confirm that the scatter in the absolute magnitudes of the galaxies is small ( 0.3 magnitudes). The BCGs exhibit very little luminosity evolution in this redshift range: if $q_{0}=0.0$ we detect no luminosity evolution; for $q_{0}=0.5$ we measure a small negative evolution (i.e., BCGs were about 0.5 magnitudes fainter at $z=1$ than today). If the mass in stars of these galaxies had remained constant over this period of time, substantial positive luminosity evolution would be expected: BCGs should have been brighter in the past since their stars were younger. A likely explanation for the observed zero or negative evolution is that the stellar mass of the BCGs has been assembled over time through merging and accretion, as expected in hierarchical models of galaxy formation. The colour evolution of the BCGs is consistent with that of an old stellar population $\left(z_{\text {form }}>2\right)$ that is evolving passively. We can thus use evolutionary population synthesis models to estimate the rate of growth in stellar mass for these systems. We find that the stellar mass in a typical BCG has grown by a factor $\simeq 2$ since $z \simeq 1$ if $q_{0}=0.0$ or by factor $\simeq 4$ if $q_{0}=0.5$. These results are in remarkably good agreement with the predictions of semi-analytic models of galaxy formation and evolution set in the context of a hierarchical scenario for structure formation. 